\title{
Evaluation of Scopolamine Patch Pharmacotherapy for Motion Sickness in S. Korean
}

\author{
Sung Cil Lim*, Myung Koo Lee, Chong Kil Lee, and Bo Reum Lee \\ College of Pharmacy, CBITRC, Chungbuk National University, 410 Sungbong-ro, Heungduk-gu, Cheongju 361-763, S. Korea
}

(Received June 13, 2008; Accepted June 21, 2008)

\begin{abstract}
Scopolamine patch is an effective anticholinergic and antiemetic agent for motion sickness in S. Korea. Since this medication is Over The Counter (OTC) medication and may cause serious side effects when misused. Therefore, we evaluate the safety and efficacy for scopolamine patch in this study. We selected and surveyed total 43 patients who purchased scopolamine patch from three different pharmacies located at $S$ and $C$ Express Bus terminals and P Port at Seoul, Cheongju, and Busan. In the result, 11 patients (8 15 year old: 25.6\%) were correctly used scopolamine patch with a children dose and 2 patients were misused. 26 (15 60 year old: $60.4 \%$ ) and 4 (over 60 year old: $9.3 \%$ ) patients were correctly used with a adult dose. 33 patients $(69.9 \%)$ answered that they knew the directions well how to use scopolamine patch correctly. Only $24.2 \%(n=8)$ patients learned the directions by pharmacist's consultation. Most patients (45 frequencies with duplicate counts) had some experienced side effects and among those drowsiness is the most common one. In conclusion, scopolamine patch as a non-prescription drug (OTC) should be monitored by pharmacist with correct drug consultation.
\end{abstract}

Keywords: motion-sickness, Scopolamine patch, OTC, consultation

\section{INTRODUCTION}

Motion-sickness is a distressing symptoms, which can caused by various transportations, such as a car, ship, airport or train, accompanied by nausea, vomiting, pallor and headaches, and it is caused by an imbalance between the cholinergic and noradrenergic parts of the central nervous system. In general, it has a major influence factor on modern traveling activities and rapidly spreading engagement in virtual reality immersion due to very unpleasant feeling for people and therefore many people frequently use anti-motion sickness agents (Parrot et al., 1989).

Scopolamine, a belladonna alkaloid, is indicated for prophylactic treatment against motion sickness. It is a very effective anticholinergic and anti-motionsickness agent which acts as a competitive inhibitor at postganglionic muscarinic receptor sites of the parasympathetic nervous system, and on smooth muscles that respond to acetylcholine but lack cholinergic innervation. However, scopolamine is associated with a high incidence of side

\footnotetext{
${ }^{*}$ Corresponding author

Tel: +82-43-261-3590, Fax: +82-43-268-2732

E-mail: slim@chungbuk.ac.kr
}

effects and some could be idiosyncratic adverse effects. Most of adverse effects are only decreasing quality of life, but some are very dangerous and those must be carefully monitored and prevented (Parrrot et al., 1989; Price et al., 1981).

Currently scopolamine patch is being used in both South Korea and the United States. In 1981, it was developed and available as a prescription-only medication in the United States. But one day the pharmaceutical company suddenly stopped the manufacturing of scopolamine due to several dangerous side effects (epilepsy and enlargement of the pupil, etc) and then re-produced again in year 2005. However it still strictly requires a doctor's prescription and pharmacist's drug consultation in order to dispense scopolamine in the U.S.A. Compared to the U.S.A., scopolamine patch as an anti-motion-sickness agent in S. Korea is released as Over The Counter (OTC: non-prescription) medication in August 1985 and so it can be easily purchased by the public. Whenever pharmacist dispense the scopolamine patch, pharmacist should provide patient drug consultations with proper directions of use and cautions. Because its misuse may casuse side effects and those should be prevented (Parrrot et al., 1989; Price et al., 1981). 
Also scopolamine patch is available as two versions of strength which are each for children only (age of 8 thru 15 year old) or for adults only (age of over 15 year old) in S. Korea. However, scopolamine patch is existed one strength dosasge form which allowed only for over 12 year old patient in the U.S.A. Especially, motion sickness is more frequently occurred in the elders and children who are weaker than adults in general. Therefore, they have greater probability to be exposed to side effects from increasing frequency of use. As we mentioned, scopolamine can cause some serious side effects when misused. It must require precise directions for usage and pharmacist's accurate drug consultation in order to improve health welfare of the people (Shupak et al., 1989).

Therefore we performed on a cross-sectional study which evaluate patient's knowledge for directions of scopolamine patch use from the purchaser, effectiveness and find out how many patients are aware of the side effects andcautions of scopolamine transdermal therapeutic system (TTS) during pharmacotherapy of motion sickness among Korean patients.

\section{General Information of Scopolamine TTS}

The chemical name of scopolamine is benzeneacetic acid 9-methyl-3-oxa-9-azatricyclo[3.3.1.0] ${ }^{2,4}$ non-7-yl ester and has low bioavailability (10.7 48.\%) because of undergoing the first bypass effect. Therefore it commonly uses as the form of patch for the prevention or treatment of motion sickness. It is available as transdermal therapeutic systems anti-motion sickness in S. Korea. Those are available which are released as $1.5 \mathrm{mg}$ for adults or $0.75 \mathrm{mg}$ for children over 72 hours per patch. The scopolamine patch contains a reservoir of scopolamine programed to deliver $0.5 \mathrm{mg}$ over a 3-day period (Zohar et al., 2000)

\section{Proper directions of use}

If the scopolamine patch is being used to prevent the nausea and vomiting of motion sickness, plan to apply one transdermal patch at least 4 hours before you need it and remove the patch after 3 days and throw it away. Do not cut the patch. Select a hairless area of skin behind one ear, taking care to avoid any cuts or irritations. Once patients have placed the patch behind ear, do not move it for as long as want to use it. After removing the patch, be sure to wash hands and the area behind ear thoroughly with soap and water. If wish to control the nausea and vomiting of motion sickness for longer than 3 days, remove the first patch after 3 days and place a new one behind the other ear. In order to avoid children or pets play around with a disposed patch, you must dispose it in a garbage can (Gorden et al., 1986; Graybiel et al., 1976; Paul et al., 2007)

\section{Side effects and cautions}

The most common side effects are dryness of the mouth and drowsiness. Temporary blurring of vision and dilation of the pupils may occur, infrequently disorientation, memory disturbances, dizziness, restlessness, hallucination, confusion, difficulty urinating, skin rash, changing heart rate, dry itch, acute dystonic reaction such as abnormal muscle spasm and posture, uncontrolled body movement, abnormal speech, reddened whites of the eyes and eye pain can happen. The patient who is pregnant or nursing woman, have (or have had) glaucoma or a predisposition to glaucoma, any metabolic, heart, liver, kidney, or other serious medical conditions, any obstruction of the stomach or intestine, difficulty in urinating due to prostate enlargement or any bladder obstruction, allergy or skin rash to any drug, especially scopolamine, or chemical or food substance, should be precautious in using transdermal patch, and persons who are hypersensitive to the scopolamine or have angle-closure (narrow angle) (Gilman et al., 1990; Gone et al., 1998; Paul et al., 2007).

\section{MATERIAL AND METHODS}

\section{Subjects and study period}

This study was performed on randomly chosen patients who purchased scopolamine patch from different three pharmacy stores at Seoul South Bus Terminal, Chung-ju Suburbs Bus Terminal, Busan Traveler Port Terminal and from August 6th thru 30th, 2007. It was designed as a cross-sectional study which was surveyed by a questionnaire and evaluated the four main parts: epidemiology of patients for the patient's epidemiological characteristics, directions of scopolamine patch use, effectiveness and side effects. There were total of 43 patients who agreed to survey and the questions were verbally asked and written by the researcher. The questionnaire was included total 14 questions and the details are in the Table I (Table I).

\section{Epidemiology of patient's characteristics}

In order to find out whether a patient used the right dose of medication or not, we evaluated a patient's gender, age, weight and height, and co-disease by question 1 thru 4 of the questionnaire (Table I).

\section{Directions of scopolamine patch use}

In order to find out a patient's knowledge of the proper directions of scopolamine use, we evaluated the reason 
Table I. Questionnaire for scopolamine patch

Place of Questionnaire: Express Bus terminal / Port

1. Gender : male / female

2. Age
(1) under 7 years
(2) $8 \sim 15$ years
(3) above 15 years

3. Height/ Weight: ( $\mathrm{cm} /$ $\mathrm{kg})$

4. Do you have any other disease currently?
(1) Hypertension
(2) Glaucoma
(4) Constipation
(5) Xerophthalmia
(3) Epilepsy
(6) $\operatorname{Etc}()$

5. Have you ever use this medication before?:

$Y$ : How often?: ( times)

$\mathrm{N}$ : What kind of reasons makes you use this medication?:

(1) Because you suffered from motion-sickness in past.

(2) For precaution against long journey

(3) Etc (

5-1. Do you know how to use this medication exactly?:

$\mathrm{Y}$ : How did you know th method?:
(1) by reading of Package insert
(2) by listening pharmacist's explanation
(3) by seeing other people use i
(4) Etc (

$\mathrm{N}$

5-2. How come do you choose this medication among many other medications?:
(1) Recommendation by pharmacist
(2) As other people using it
(3) By commercial
(4) Etc ( )

5-3. Without this medication, how severe is motion-sickness during using transprtation? :
(1) A little bit
(2) Little bit of feeling motion-sickness(tolerable)
(3) Feeling of motion-sickness
(4) Strong feeling of motion-sickness
(5) Very strong feeling of motion-sickness(intolerable)

6. When do you apply this medication(patch) on your body preliminary to start your journey?
(1) Just prior to start
(2) 1 3 hours before
(3) $4 \sim 12$ hours before
(4) A day before

7. When do you usually detach the patch?:

(1) Before arriving(in the middle journey)

(2) As soon as arrive

(3) ( )hours past after arriving

(4) When feeling of motion-sickness stops

(5) Any time

7-1. What is the longest using time?: ( hours)

8. What is the usual transportation time using this medication?:
(1) Less than 2 hours
(2) 2 4 hours
(3) 4 6 hours
(4) $6 \sim 12$ hours
(5) Longer than 12 hours

9. When you use this medication in the past, did you have any adverse effect?:
$Y$ : (1) Blurred vision
(2) Oral dryness
(3) Drowsiness
(6) Difficulty urination
(4) Nervous, anxious
(5) Skin allergy on attach area
(9) Salivation
(7) Headache
(8) Allergic reaction
(11) Etc (
(10) Eye dryness

$\mathrm{N}$

10. Do you have any other medication taking currently?:
(1) Cold medicine
(2) Vitamin supplements
(4) Healthy supplying food(
(5) Anti hypertensive agent )
(8) Anti glaucoma medicine
(6) Stool softeners
(3) Anti epileptic agent
(7) Anti headache medicine 
for choosing particular medication, average frequency of dosage, a type of transportation that the patient is using, period of using that transportation, a method of installation of the medication and time for application, and subjective's opinion for the level of sickness by question 5 thru 8 of the questionnaire (Table I).

\section{Effectiveness for anti- motion sickness}

We evaluate the effectiveness of anti-motion sickness for scopolamine patch totally by pt's subjective opinion such as sickness feeling or occurrence of nausea or vomiting, etc. If the patient think scopolamine patch made them feel comfortable compared to not use it, they can choose their answer whatever they judge it. It was evaluated by question 5-3 of the questionnaire (Table I).

\section{Side effects and cautions}

We evaluated patient's side effects by choosing example of question 9 when patient had experienced any side effects with application of scopolamine. And we also evaluated co-medication and co-diseases for possible drug drug or disease interactions. Also we evaluate if side effects or interaction are existed and then how dangerous those side effects were and how often they occur.

\section{Statistics}

This study is designed by a cross-sectional study model with a questionnaire. In this time, the collected data and deviations are indicated and compared in percentile simply by using Microsoft Excel program.

\section{RESULTS}

\section{Epidemiology of patient's characteristics}

From August 6th to 30th of 2007, 19 among 43 patients were male and 24 patients were female. 4 patients were purchased scopolamine patch for ship travel and 39 patients for express-bus travel. 2 patients under age of 8 year old were used scopolamine patch that they should not allowed to use scopolamine patch and pharmacist should not dispense them (4.7\%). 11 patients were between age of 8 and 15 year old and they used scopolamine patch for children dose (25.6\%). Most patients who 26 patients between age of 15 and 60 year old $(60.4 \%)$ and 4 patients over age of 60 year old $(9.3 \%)$ were also used scopolamine patch for adult dose. Data of weight and height for relevant dose evaluation were collected and none of the patients were highly over-weighted or under-weight (Table II).

\section{Proper directions of scopolamine patch use}

33 patients $(69.9 \%)$ answered that they know the directions of scopolamine well that is how to use the medication correctly. The ways of how the patients know the direction were by pharmacist's consultation ( 8 patients, $24.2 \%$ ), reading of package insert (11 patients, $33.3 \%$ ), and from watching of the peers' usage (14 patients, $42.4 \%$ ). And 10 patients do not know at all (10 patients, $23 \%$ ). One patient (2.3\%) answered that scopolamine patch was installed a day before departing time, 9 patients $(20.9 \%)$ installed four to twelve hours before departure, 23 patients $(53.5 \%)$ installed one to three hours before departure, and 10 patients $(23.3 \%)$ installed the patch right before departure. 34 patients (79.1\%) removed patch as soon as they arrived, and the rest 9 patients $(20.9 \%)$ removed the patch whenever they remembered. Appling time were usually under 8 hours (60.1\% patients). $4.6 \%$ patients applied the patch over 24 hours even though finishing the transportation. 59.8\% patients use scopolamine patch for 2 thru 4 hours as

Table II. Demographic characteristics of patients

\begin{tabular}{|c|c|c|c|}
\hline \multicolumn{2}{|r|}{ Parameter } & Frequency of use in the past (n) & Percentage (\%) \\
\hline \multirow{2}{*}{ Sex } & Male & 19.0 & 44.2 \\
\hline & Female & 24.0 & 55.8 \\
\hline \multirow{4}{*}{$\begin{array}{c}\text { Age } \\
\text { (year) }\end{array}$} & Under 8 & 2.0 & 4.7 \\
\hline & $8 \sim 15$ & 11.0 & 25.6 \\
\hline & $15 \sim 60$ & 26.0 & 60.4 \\
\hline & Over 60 & 4.0 & 9.3 \\
\hline \multirow{2}{*}{ Dose } & $\begin{array}{c}\text { For adult only use } \\
\text { (age over } 15 \text { year old: } 1.5 \mathrm{mg} / 1 \text { sheet) }\end{array}$ & 13.0 & 30.2 \\
\hline & $\begin{array}{l}\text { For children treatment } \\
\text { (age of 8 15 years: } 0.75 \mathrm{mg} / 1 \text { sheet) }\end{array}$ & 30.0 & 69.8 \\
\hline
\end{tabular}


transportation time and $34.5 \%$ patients use it for 4 thru 6 hours as transportation time. Only $4.6 \%$ patients use it for 6 hours of transportation time. Also, $79.1 \%$ patients

Table III. Analysis for usage of scopolamine patch

\begin{tabular}{|c|c|c|c|}
\hline \multicolumn{2}{|c|}{ Parameter } & $\begin{array}{l}\text { Frequency } \\
\text { (n) }\end{array}$ & $\begin{array}{c}\text { Percentage } \\
(\%)\end{array}$ \\
\hline \multirow{2}{*}{ Recognition } & No & 10.0 & 23.0 \\
\hline & Yes & 33.0 & 69.9 \\
\hline \multirow{3}{*}{ Route } & $\begin{array}{c}\text { Patient } \\
\text { consultation }\end{array}$ & 8.0 & 24.2 \\
\hline & Package insult & 11.0 & 33.3 \\
\hline & Other people & 14.0 & 42.4 \\
\hline \multirow{7}{*}{$\begin{array}{l}\text { Maximum } \\
\text { Period of } \\
\text { Usage }\end{array}$} & 2 4 hrs & 6.0 & 13.8 \\
\hline & $4 \sim 6 \mathrm{hrs}$ & 11.0 & 23.3 \\
\hline & $6 \sim 8 \mathrm{hrs}$ & 10.0 & 23.0 \\
\hline & $8 \sim 12 \mathrm{hrs}$ & 3.0 & 6.9 \\
\hline & $12 \sim 16 \mathrm{hrs}$ & 1.0 & 2.3 \\
\hline & 16 24 hrs & 0.0 & 0.0 \\
\hline & Over 24 hrs & 2.0 & 4.6 \\
\hline \multirow{4}{*}{$\begin{array}{c}\text { Point of } \\
\text { Installment }\end{array}$} & 1 days before & 1.0 & 2.3 \\
\hline & $4 \sim 12 \mathrm{hrs}$ before & 9.0 & 20.9 \\
\hline & 1 3 hrs before & 23.0 & 53.5 \\
\hline & $\begin{array}{l}\text { just before } \\
\text { departure }\end{array}$ & 10.0 & 23.3 \\
\hline \multirow{2}{*}{$\begin{array}{l}\text { Point of } \\
\text { Removal }\end{array}$} & At arrival & 34.0 & 79.1 \\
\hline & Whenever & 9.0 & 20.9 \\
\hline \multirow{2}{*}{$\begin{array}{c}\text { Type of } \\
\text { transportation }\end{array}$} & by bus & 39.0 & 87.9 \\
\hline & by ship & 4.0 & 12.1 \\
\hline \multirow{5}{*}{$\begin{array}{l}\text { Reason of } \\
\text { selection }\end{array}$} & Commercial & 17.0 & 39.1 \\
\hline & Other's use & 20.0 & 46.0 \\
\hline & Past experiences & 4.0 & 9.2 \\
\hline & $\begin{array}{l}\text { Pharmacist's } \\
\text { recommendation }\end{array}$ & 1.0 & 2.3 \\
\hline & Others & 1.0 & 2.3 \\
\hline \multirow{5}{*}{$\begin{array}{l}\text { Using numbers } \\
\text { over last } 1 \text { year }\end{array}$} & Under 1 & 11.0 & 23.3 \\
\hline & $1 \sim 2$ & 14.0 & 32.2 \\
\hline & $3 \sim 4$ & 10.0 & 23.0 \\
\hline & $5 \sim 6$ & 5.0 & 11.5 \\
\hline & Over 7 & 3.0 & 6.9 \\
\hline \multirow{4}{*}{$\begin{array}{l}\text { Time for } \\
\text { transportation }\end{array}$} & Under 2 hours & 0.0 & 0 \\
\hline & $2 \sim 4$ hours & 26.0 & 59.8 \\
\hline & $4 \sim 6$ hours & 15.0 & 34.5 \\
\hline & $6 \sim 12$ hours & 2.0 & 4.6 \\
\hline
\end{tabular}

removed the patch at time of arrival but $20.9 \%$ patient were removed the patch whenever they want and it will be dangerous for the health. During the last 1 year, 25 patients $(55.5 \%)$ were experienced for use of scopolamine patch under 2 times and even though 3 patients (6.9\%) were used over 7 times. Most of reasons for selection of scopolamine patch were commercial advertisement $(39.1 \%)$ or other's recommendation $(46.0 \%)$ and only 1 patient was by pharmacist's recommendation (2.3\%) (Table III).

\section{Effectiveness of scopolamine patch}

2 patients $(2.6 \%)$ use medication even though there is no sign of motion sickness and 18 patients (41.4\%) experienced very little bit of motion sickness, 13 patients (29.9\%) experienced some motion sickness, 2 patients

Table IV. Side effects of scopolamine patch

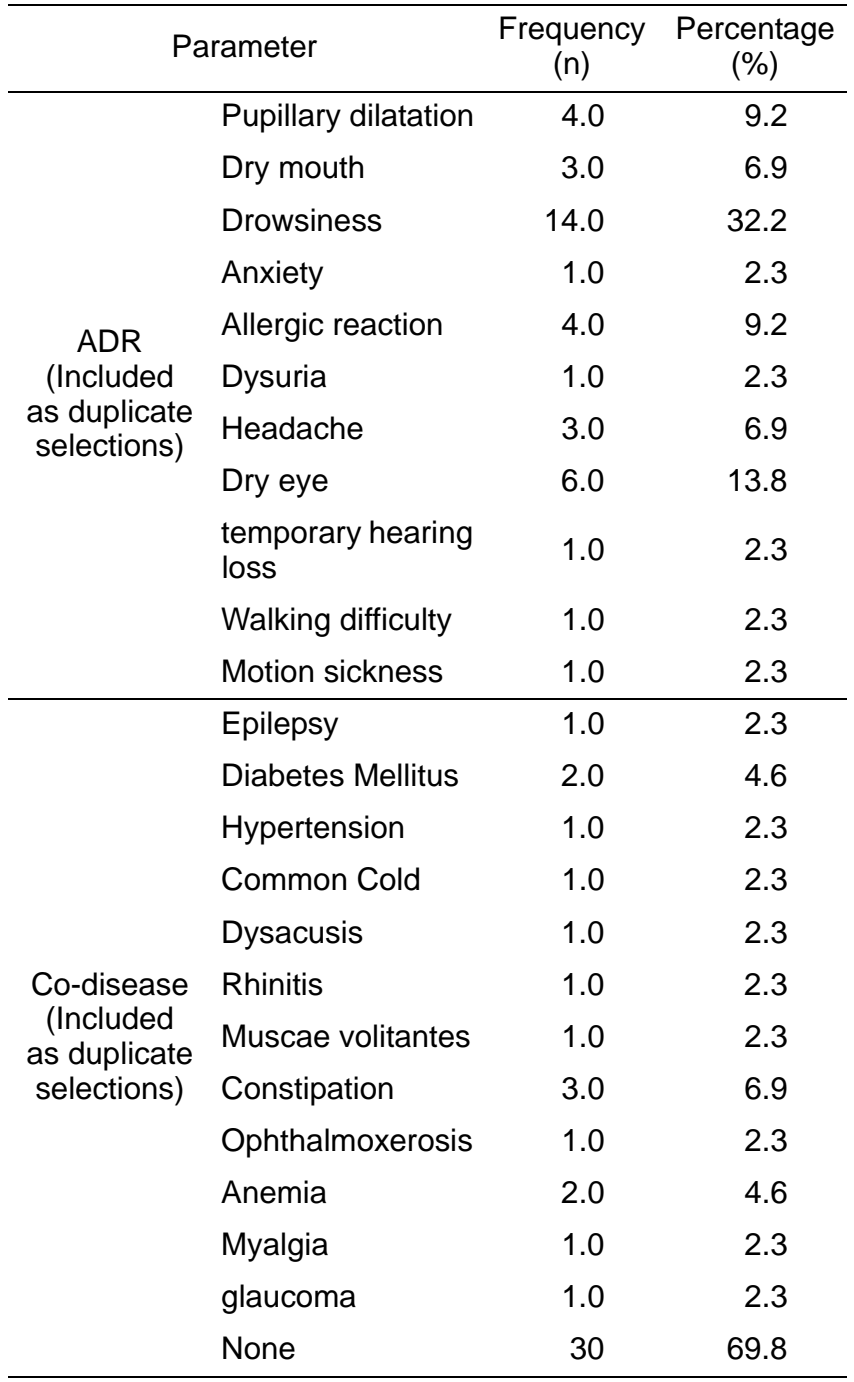




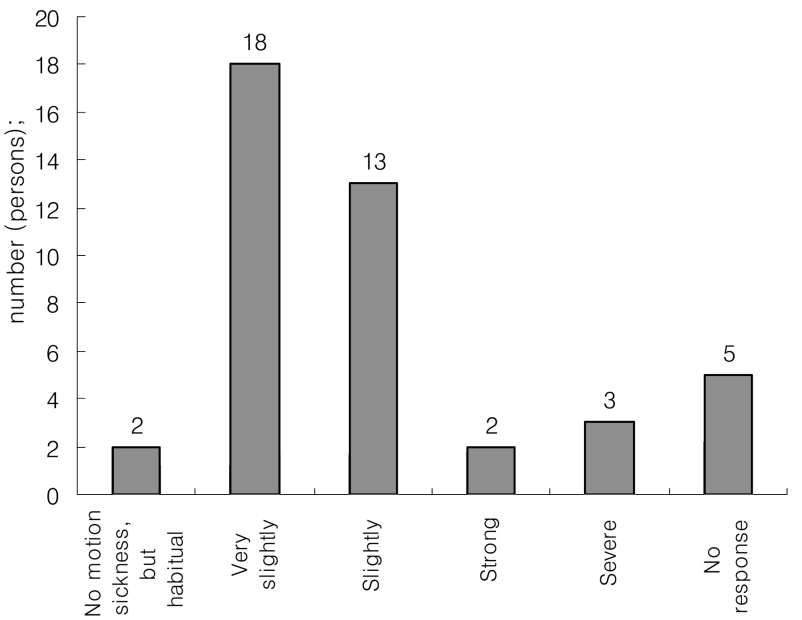

Fig. 1. Degree of motion sickness by patient's decision for purchasing scopolamine patch before using a transportation : 2 patients $(2.6 \%)$ purchased scopolamine patch even though they had no sign of motion sickness in the past. 18 patients $(41.4 \%)$ experienced very little bit of motion sickness, 13 patients $(29.9 \%)$ experienced some motion sickness, 2 patients $(4.6 \%)$ experienced intense sickness, 3 patients $(6.9 \%)$ experienced little intense sickness, and 5 patients (11.5\%)experienced an extreme motion sickness in the past and therefore they purchased scopolamine patch (39 patients for a bus and 4 patients for a ship transportation). In Fig. 1, 'levels of motion sickness' corresponds to level of vomiting and 'little bit of motion sickness' indicates possibility of misjudge and the state which the patient can hold on but vomiting does not arise at all.

(4.6\%) experienced intense sickness, 3 patients $(6.9 \%)$ experienced little intense sickness, and 5 patients (11.5\%) experienced an extreme motion sickness. One patient still had motion sickness with application of scopolamine patch (Fig. 1)(Table IV).

\section{Side effects and cautions}

Most patients (45 frequencies with duplicate count) had experienced side effects and drowsiness is the most common side effects. Occurring side effects were pupillary dilatation (4 patients, 9.2\%), dry mouth (3 patients, $6.9 \%$ ), drowsiness (14 patients, $32.2 \%$ ), anxiety (1 patient, $2.3 \%$ ), allergic reaction (4 patients, 9.2\%), dysuria (1 patient, $2.3 \%)$, headache (3 patients, $6.9 \%)$, dry eye (6 patients, $13.8 \%$ ), temporary hearing loss (1 patient, $2.3 \%$ ), difficult walking (1 patient, 2.3\%), and no effect (1 patient, $2.3 \%$ ). Some patients (15 patients) experienced multiple of these symptoms. 13 patients had co-disease such as epilepsy ( 1 patient, $2.3 \%$ ), diabetes (2 patients, $4.6 \%$ ), hypertension (1 patient, 2.3\%), common cold (1 patient, $2.3 \%)$, hearing impairments (1 patient, $2.3 \%)$, rhinitis (1 patient, $2.3 \%$ ), muscae volitantes (1 patient, $2.3 \%)$, mus- cle pain (1 patient, 2.3\%), constipation (3 patients, $6.9 \%$ ), xerophthalmia (1 patient, 2.3\%), glaucoma (1 patient, $2.3 \%$ ), anemia (2 patients, 4.6\%)(Table IV).

\section{DISCUSSION}

This research was performed on total 43 travelers who purchased scopolamine patch as an anti-motion-sickness medication at three different pharmacy stores from August 6th to 30th of 2007. Scopolamine patch is a popular and effective anticholinergic and antiemetic agent for motion sickness in S. Korea. However, since this medication is OTC product and can cause serious side effects and problems such as seizure or dilation of pupil when misuses. And especially the scopolamine patch has been applied by the age limitation for use of a anti-motion sickness agent in the U.S.A (McEvoy et al., 1990; Nachum et al., 2006). Therefore we evaluate the patient's age range, the pattern and reason of selection, dosing method, side effects and effectiveness when use a scopolamine patch as a anti-motion sickness in S. Korea by a questionnaire in order to prevent misusing or overusing from the medication in the sight of pharmacist's view.

All 13 patients under 15 year old were applied scopolamine patch for children use only (for age of 8 thru 15 year). However among them two patients with age of 4 and 7 year old were used each scopolamine patch who should not be allowed. Therefore, whoever those pharmacists must not be dispensed those patients with consultation and should prevented. The most patients use the scopolamine patch average 1 or 2 times per year. However some patients (18.4\%) were excessively use it over 5 times per year and they should need to monitor carefully or suggestion of a other method for preventing of motion sickness. $69.9 \%$ patients answered they knew the directions well. However, in the results, only 53.5\% patients knew correctly for the application or removing time. $20.9 \%$ patient were removed the patch whenever they want which should not be allowed. Especially, only $24.2 \%$ patients got the directions of use from the pharmacist's consultation. Therefore, they must be re-educate by pharmacist's consultation in the future. Most side effects were related to anticholinergic reactions such as drowsiness, dryness, dysuria (Attis et al., 1987; Paul et al., 2007). However, some side effects such as temporary hearing loss were dangerous and must be prevented with monitoring. One patient had experienced motion sickness with application of scopolamine patch and the patient should be monitored the reason. Some problems were produced during the this study. First of all, There 
were some patients or parents of young age-patients who were not perfectly sure about the medical backgrounds of their patients which is very important for the safe use of this medication in order to prevent side effects or interactions. Also, even though they knew exactly about their medical backgrounds, there may had been some questions that they did not or did not want to answer precisely because this medication usually use in the hectic situation such as terminal or port. Especially, some youth patients did not know their exact weight or height. Because of the characteristics of the place where the surveys were taken, it was difficult to gather enough data in such limited times. However, in the result of this study, some age groups (4 year and 7 year old patients) who should not be allowed but were misused this medication. for a anti-motion sickness. Also, this age group purchased their medication by their parents and it could be produced some problems or benefit. Many patients misunderstood they know the proper directions which is not true. Also some teenagers (4 patients) purchased the scopolamine patch that they did not cause motion sickness when they use a Express bus or ship. That is the reason being of the selection of a scopolamine patch was because they wanted to install the patch together with one of friend and for fun which is very dangerous situation.

Overall, for the characteristics of the OTC medications which do not require a prescription, anybody can purchase the OTC medication. Sometimes, it can bring dangerous situation for some patients and eventually affect patient's health in a wrong way. Therefore, all pharmacists should monitor all medications including of OTC products when they dispense and they must provide a drug consultation to the patient in order to prevent any problems from misusing or wrong application of the medication.

\section{ACKNOWLEDGEMENTS}

"This work was supported by the Korea Research
Foundation Grant funded by the Korean Government (MOEHRD)" (The Regional Research Universities Program/Chungbuk BIT Research-Oriented University Consortium).

\section{REFERENCES}

Attias, J., Gordon, C., Ribak, J., Binah, O., and Rolnick, A. (1987). Efficacy of transdermal scopolamine against seasickness: A 3-day study at sea. Aviat Space Environ Med. 58, 6062.

Gonen, A., Gordon, C. R., Spitzer, O., Shahal, B., and Shupak, A. (1998). Comparison of performance under the influence of three anti-motion sickness drug. Aviat Apace Environ Med. 69, 209.

Gilman, A. G. et al. (ed.) (1990). The Pharmacological Basis of Therapeutics (8th Ed.); Pergamon Press, New York, NY. 150165.

Gorden, C., Binah, O., Attias, J., and Rolnick, A. (1986). Transdermal scopolamine: Human performance and side effects. Aviat Apace Environ Med. 57, 236-240.

Graybiel, A., Knepton, J., and Shaw, J. (1976). Prevention of experimental motion sickness by scopolamine absorbed through the skin. Aviat Apace Environ Med. 47, 1096-1100.

Hornby, P. J. (2001) Central neurocircuitry associated with emesis. Am J Med. 111, 106-12.

McEvoy, G. K. (8ed.) (1990). AHSF Drug Information; American Society of Hospital Pharmacists, Bethesda, MD. 608-611.

Nachum, Z., Shupak, A., and Gordon, C. R. (2006). Transdermal scopolamine for prevention of motion sickness. Clinical pharmacokinetics and Therapeutic Applications. 45(6), 54366.

Parrott, A. C. (1989). Transdermal scopolamine: A review of its effects upon motion sickness, Psychological performance, and physiological functioning. Aviat Apace Environ Med. 60, 1-9.

Price, N. M., Schmitt, L. G., McGuire, J., Shaw, J. E., and Trobough, G. (1981). Transdermal scopolamine in the prevention of motion sickness at sea. Clin Pharmacol Ther. 29, 414-419.

Shupak, A., Gordon, C. R., Spitzer, O., Mendelowitz, N., and Melamed, Y. (1989). Three years' experience of transdermal scopolamine: Long-term effectiveness and side-effects. Pharmatherapeutica. 5, 365-370 